\title{
BMJ Open Allogeneic adipose tissue-derived mesenchymal stem cells in ischaemic stroke (AMASCIS-02): a phase IIb, multicentre, double-blind, placebo- controlled clinical trial protocol
}

Elena de Celis-Ruiz, ${ }^{1}$ Blanca Fuentes (D) , ${ }^{1}$ Francisco Moniche, ${ }^{2}$ Joan Montaner, ${ }^{3}$ Alberto M Borobia, ${ }^{4}$ Maria Gutiérrez-Fernández, ${ }^{1}$ Exuperio Díez-Tejedor ${ }^{1}$

To cite: de Celis-Ruiz E, Fuentes B, Moniche F, et al. Allogeneic adipose tissuederived mesenchymal stem cells in ischaemic stroke (AMASCIS-02): a phase IIb, multicentre, double-blind, placebo-controlled clinical trial protocol. BMJ Open 2021;11:e051790. doi:10.1136/ bmjopen-2021-051790

- Prepublication history and additional supplemental material for this paper are available online. To view these files, please visit the journal online (http://dx.doi.org/10.1136/ bmjopen-2021-051790).

EdC-R and BF contributed equally.

Received 29 March 2021 Accepted 28 July 2021

D) Check for updates

(c) Author(s) (or their employer(s)) 2021. Re-use permitted under CC BY-NC. No commercial re-use. See rights and permissions. Published by BMJ.

For numbered affiliations see end of article.

\section{Correspondence to}

Dr Blanca Fuentes;

blanca.fuentes@salud.madrid. org

\section{ABSTRACT}

Introduction Stroke is a serious public health problem, given it is a major cause of disability worldwide despite the spread of recanalisation therapies. Enhancement of brain plasticity with stem cell administration is a promising innovative therapy to reduce sequelae in these patients. Methods and analysis We have developed a phase $\mathrm{lb}$, multicentre, randomised, double-blind, placebo-controlled clinical trial protocol to evaluate the safety and efficacy of intravenous administration of allogeneic adipose tissue-derived mesenchymal stem cells (AD-MSCs) in patients with acute ischaemic stroke, concurrently with conventional stroke treatment. Thirty patients will be randomised on a 1:1 basis to receive either intravenous placebo or allogeneic AD-MSCs as soon as possible within the first 4 days from stroke symptom onset. Patients will be followed up to 24 months after randomisation. The primary objective is the safety assessment of early intravenous administration of allogeneic AD-MSCs by reporting all adverse events and neurological or systemic complications in both treatment groups. Secondary objectives assess efficacy of early intravenous AD-MSC treatment in acute ischaemic stroke by evaluating changes in the modified Rankin Scale and the National Institutes of Health Stroke Scale throughout the follow-up period. In addition, brain repair biomarkers will be measured at various visits. Ethics and dissemination This clinical trial has been approved by the Clinical Research Ethics Committee of La Paz University Hospital (Madrid, Spain) and by the Spanish Agency of Medication and Health Products and has been registered in Eudra CT (2019-001724-35) and ClinicalTrials.gov (NCT04280003). Study results will be disseminated through peer-reviewed publications in Open Access format and at conference presentations.

\section{INTRODUCTION}

Stroke is a serious health problem, given it is one of the most common causes of mortality and the leading cause of disability due to neurological diseases worldwide. In 2016, the global estimated incidence of stroke ranged between 189 and 218 cases
Strengths and limitations of this study

- This is the first multicentre clinical trial evaluating safety and possible efficacy of early intravenous treatment with adipose-derived mesenchymal stem cells in ischaemic stroke.

- The trial is designed as a randomised, placebocontrolled, double-blind study, so biases will be minimised, albeit limiting general population applicability.

- Limitations for external validity are the fact that patients are recruited in a single country from only two centres and the small sample size.

per 100000 inhabitants. ${ }^{1}$ A large proportion of patients with ischaemic stroke have persistent neurological deficits that impair their daily life activities, and many also experience non-neurological complications, such as pulmonary and urinary tract infections, deep vein thrombosis and depression. In the last two decades, there has been a breakthrough in ischaemic stroke treatments. However, currently available therapies, such as intravenous thrombolysis and mechanical thrombectomy, focus only on reperfusion in the acute phase and are not accessible to all patients due to a narrow time window and eligibility issues. Furthermore, these cerebral reperfusion-based therapies cannot reverse established ischaemic injury or reduce its sequelae. Therefore, to reduce the burden of this disease, there is an urgent need for therapies that can enhance patient recovery after stroke.

After a brain injury, several interrelated mechanisms, such as neurogenesis, gliogenesis, oligodendrogenesis, synaptogenesis and angiogenesis take place to repair the damaged tissue. In clinical practice, these processes are 
stimulated through rehabilitation therapies; however, cell therapies promote tissue repair exogenously, ${ }^{2}$ which could complement current reperfusion treatments for acute ischaemic stroke.

Stem cells are immature cells with the capacity to differentiate into diverse cell lines. At present, challenges concerning the most suitable cell type, route and time of administration are the main issues for progression from preclinical studies to the design of clinical trials. ${ }^{3-5}$

Regarding the route of administration, preclinical studies have shown that it is not mandatory for stem cells to migrate and nest at the injury site to achieve functional recovery after stroke, given their therapeutic effect is also due to secretion of trophic factors and modulation of the immune response. ${ }^{56}$ Intravenous administration has been proven to be non-inferior to other routes in preclinical studies, such as in intracerebral, intrathecal or intraarterial deliveries, and it is far less invasive. Regarding cell type, preclinical and initial clinical trial data ${ }^{57-11}$ suggest that mesenchymal stem cells (MSCs) constitute a safe and possibly effective therapy in ischaemic stroke. Allogeneic MSCs lack human leucocyte antigen-class II molecules, avoiding the risk of rejection and allowing short timings of administration.

Most studies concerning cell therapies in ischaemic stroke have been performed in chronic stages of the disease, although data from preclinical studies as well as the recent MASTERS trial suggest their early administration could be more effective. In the MASTERS trial, ${ }^{12}$ intravenous administration of multipotent adult progenitor cells in patients with acute ischaemic stroke did not provoke any safety concerns or administration issues, and a tendency towards better functional recovery in the early treatment arm ( $<36$ hours from symptom onset instead of $36-48$ hours) versus placebo was observed. This observation was also supported by the fact that the trophic factors released during the acute phase after stroke inhibited the first steps of the ischaemic cascade and enhanced brain protection mechanisms. ${ }^{3} 1314$

Adipose tissue-derived mesenchymal stem cells (ADMSCs) are abundant, accessible and easy to obtain by lipoaspiration techniques; ${ }^{15}{ }^{16}$ they also can be administered without ethical concerns. ${ }^{13}$ Their intravenous administration has been proven safe in rat models of ischaemic stroke and has also been associated with good functional recovery, reductions in cell death and increased cell proliferation at the peri-infarct zone without observed implantation of the AD-MSCs at the infarct site. ${ }^{1718}$ To our knowledge, the first clinical trial assessing the safety and efficacy of intravenous administration of allogeneic AD-MSCs in patients with ischaemic stroke is the AMASCIS-01 trial (NCT01678534), ${ }^{19}$ which has been performed by our study group. Although the 2-year follow-up results have not yet been published, a safety interim analysis at 6 months showed no safety concerns when the AD-MSCs were administered within the first 2 weeks after symptom onset. ${ }^{20}$ Other case reports and clinical trials exploring this cell type in non-ischaemic neurological diseases ${ }^{21}$ and non-neurological diseases, such as in acute respiratory distress and refractory rheumatoid arthritis, have also shown no safety issues compared with the placebo group. ${ }^{22} 23$

In preclinical studies, the MSC dose in ischaemic stroke animal models has been variable, being between $10^{4}$ and $4.3 \times 10^{7}$ MSCs $/ \mathrm{kg}^{7-10}$ Results appear more beneficial for lower rather than higher cell doses, which could be due to the fact that higher doses could potentially provoke a brain embolus or slow cerebral blood flow. ${ }^{9}$ Our study group has used doses of $1-2 \times 10^{6}$ cells $/ \mathrm{kg}$ in preclinical studies without issue. In the MASTERS trial, a single intravenous total dose of 4 or $12 \times 10^{8}$ multipotent adult progenitor cells did not cause any safety issues. In the AMASCIS-01 trial performed by our study group, a single dose of $10^{6} \mathrm{AD}-\mathrm{MSCs} / \mathrm{kg}$ was administered intravenously, also without safety concerns.

Therefore, in the AMASCIS-02 trial, we aim to show the safety of early allogeneic AD-MSC intravenous administration $\left(10^{6} \mathrm{cells} / \mathrm{kg}\right.$ of the patient's weight within the first 4 days from stroke onset) and explore the possible efficacy of this therapy in reducing stroke-associated disability.

\section{METHODS AND ANALYSIS \\ Design}

The Allogeneic Adipose Tissue derived Mesenchymal Stem Cells in Ischemic Stroke (AMASCIS-02) study is an academic, randomised, double-blind, placebo-controlled multicentre clinical trial. The two recruiting centres are La Paz University Hospital and Virgen del Rocío University Hospital, both of them in Spain.

Recruitment began in January 2021, and the estimated end date is July 2023.

\section{Primary objective}

To assess the safety of intravenous administration of allogeneic AD-MSCs within the first 4 days from stroke onset in patients with acute ischaemic stroke.

\section{Secondary objective}

To assess potential efficacy of allogeneic AD-MSCs when administered within the first 4 days from stroke onset in patients with acute ischaemic stroke.

\section{Eligibility}

Patients will be selected from the Neurology Department of both of the study centres. Acute stroke management of all patients will follow standard of care. Reperfusion therapies such as intravenous fibrinolysis and mechanical thrombectomy are allowed prior to patient screening and randomisation as long as all study inclusion and no exclusion criteria are met after their application. Inclusion and exclusion criteria are as follows:

\section{Inclusion criteria}

1. Men and women aged older than 18 years with acute ischaemic stroke. 
2. Treatment within 4 days $( \pm 1$ day) from the onset of stroke symptoms or from the last time observed as asymptomatic.

3. A CT or MRI scan compatible with the clinical diagnosis of acute non-lacunar ischaemic stroke in the region of the middle cerebral artery.

4. A score on the National Institutes of Health Stroke Scale (NIHSS) of 8-20, with at least 2 of these points in sections 5 and 6 (motor deficit) at the time of inclusion. NIHSS evaluation for screening of these patients will take place after finalisation of reperfusion therapies (if they have been performed) providing that the clinical condition of the patient is stable with no prevision of immediate recovery. A measurable focal neurological disability must persist to the time of treatment administration.

5. A prestroke score on the modified Rankin Scale $(\mathrm{mRS}) \leq 1$ (no significant disability).

6 . Women with non-childbearing potential must have either (at least one criterion):

- undergone a hysterectomy and/or bilateral oophorectomy;

- have medically confirmed ovarian failure or

- achieved postmenopausal status: cessation of regular menses for at least 12 consecutive months with no alternative cause.

7. Women with childbearing potential need a negative pregnancy test and must agree to use adequate contraception during the duration of the study.

8. Signed informed consent.

\section{Exclusion criteria}

1. Comatose patients ( $\geq 2$ score on item 1a of the NIHSS, related to degree of awareness).

2. Evidence on neuroimaging of brain tumour, cerebral oedema with midline shift and a clinically significant compression of ventricles, cerebellar or brainstem infarction and intraventricular, intracerebral or subarachnoid haemorrhage. Small petechial haemorrhages are not exclusion criteria.

3. Current drug or alcohol dependence.

4. Active infectious disease, including HIV and hepatitis $\mathrm{B}$ and $\mathrm{C}$. A controlled infection is not an exclusion criterion.

5. Pre-existing dementia.

6. A health status, clinical condition or other characteristics that preclude appropriate diagnosis, treatment or follow-up in the trial.

7. Inability or unwillingness of the individual or their representative to provide written informed consent.

8. Participation in another clinical trial.

\section{Randomisation}

Each patient will have a unique number assigned sequentially as they enter the study. The randomisation sequence was created using SAS V.9.4 statistical software (procedure 'PROC PLAN') with a 1:1 allocation. No randomisation seed was specified. The randomisation seed was generated taking the hour of the computer when the programme was executed. Randomisation will be stratified by age ( $<65$ years or $\geq 65$ years). After verifying the inclusion and exclusion criteria, patients will be assigned the study drug or a placebo solution.

\section{Masking}

The study is double-blind. After a patient is randomised by a blinded member of the research team, a non-blinded research member will request the corresponding study medication according to the randomisation code. The study medication and the placebo solution will be indistinguishable, and masking will be made by the nonblinded research team members.

\section{Outcomes}

\section{Principal variables}

The safety of AD-MSCs will be assessed using the following parameters:

- Adverse events (AEs) reported spontaneously by the patient or in response to questions not addressed, and serious AEs (death, life-threatening events, events that require inpatient hospitalisation or prolongation of existing hospitalisation, events that result in persistent or significant disability, congenital anomalies or any other event that does not meet the definitions above but can be considered potentially serious).

- Neurological and systemic complications (including abnormal laboratory values): deteriorating stroke, stroke recurrences, brain oedema, seizures, haemorrhagic transformation, respiratory infections, urinary tract infections, deep venous thrombosis and pulmonary embolism.

\section{Secondary variables}

The efficacy of intravenous treatment with AD-MSCs will be assessed using the following parameters:

- mRS: The outcome will be considered positive when the patient obtains a score of $0-3$, measured at months 3, 6, 12 and 24. An additional exploratory efficacy analysis will consider mRS shift at months 3, 6, 12 and 24.

- NIHSS: Measured at all scheduled visits. A successful outcome will occur with an improvement of $75 \%$ or more from the baseline score. An additional exploratory efficacy analysis will measure differences in the median (IQR) distribution and in the frequency of an NIHSS $\leq 1$ between groups.

- Biomarker measurement: Granulocyte-macrophage colony-stimulating factor, platelet-derived growth factor BB, brain-derived neurotrophic factor, vascular endothelial growth factor, transforming growth factor 1 , endostatin, glial fibrillar acid protein, myelin basic protein, matrix metalloproteinase 3 and extracellular vesicles will be measured at baseline, day 7 and month 3 after treatment administration. 


\section{Study procedures}

After initial screening and randomisation (visit 1) takes place, the study treatment will be administered within the first 4 days since stroke symptom onset (visit 2). Eight more visits will take place during the 24 month follow-up period (2 hours, 24 hours, 7 days or hospital discharge, $3,6,12,18$ and 24 months since treatment administration) to assess security and efficacy measures (see table 1 for details of study visits and procedures and figure 1 for schematic flowchart of the clinical trial design).

\section{Treatment or intervention}

All study medications will be industrially manufactured, tested and released according to current Good Manufacturing Practice Guidelines by Histocell. The experimental drug is an intravenous solution of allogeneic AD-MSCs in a concentration of 10 million cells per millilitre, obtained from a donor by in vitro cell culture techniques, conditioned in cryovials of Type I sterile borosilicate glass (with ringer lactate, glucosaline, sodium bicarbonate and human albumin $20 \%$ as excipients) and cryopreserved at $-150^{\circ} \mathrm{C}$. The manufacturing process is detailed in the investigator's brochure, which has been approved by the Spanish Agency of Medicines and Health products. The placebo solution has the same appearance as the study drug and is formed by its excipients. Medication vials containing either solution will be dissolved in $50 \mathrm{~mL}$ of physiological saline solution and dispensed as a single intravenous drop administration in approximately $30 \mathrm{~min}$, at a dose of 1 million cells per $\mathrm{kg}$ of the patient's weight (for the AD-MSCs).

\section{Discontinuation of study medication}

The study medication must be discontinued if a suspected anaphylactic reaction or serious $\mathrm{AE}$ occurs during its administration of if patient participation consent is withdrawn.

\section{Standard treatments for the management of acute stroke}

All patients will be managed according to current guidelines for acute stroke management, including treatment with recanalisation therapies such as intravenous thrombolysis, mechanical thrombectomy or both.

\section{Data collection and outcome measures}

An electronic case report form has been designed using MACRO. This system will maintain patient anonymity, and the data will be transferred to a '*.csv' file to analyse it with R software (V.3.5.2 or newer). To ensure the quality of the data, data management will be performed by the Spanish Clinical Research Network (SCReN). The data management plan has been approved by the principal investigator and the sponsor. Data collection forms will be included in the final report.

\section{Sample size estimates}

Given this is a pilot phase II clinical trial focused on the assessment of safety, no systematic sample size calculation applies. Based on the previous clinical trial, AMASCIS-01, it seems feasible to recruit 30 patients between the two participating centres.

\section{Statistical analyses}

Demographic and clinical characteristics will be summarised in terms of means and $\mathrm{SD}$, median and IQR or relative frequency as appropriate for each variable type. A univariate approach will be employed for calculating non-parametric tests for the continuous variables (Wilcoxon rank sum), and Fisher's or a $\chi^{2}$ test will be employed for the categorical variables. Given the binary nature of the primary outcome, a logistic regression under the minimum Akaike information criterion using all available variables will be fitted to the data, estimating ORs and their CIs. Rules of prediction for the binary outcome will be performed using receiver operating characteristic curves and k-fold cross-validation procedures. Only $\mathrm{p}$ values less than 0.05 will be considered significant. Statistical computations will be calculated using the statistical computing environment $\mathrm{R}$. The statistical analysis will be performed by the personnel from the Clinical Pharmacology Service and Central Unit of Clinical Investigation and Clinical Trials. The safety analysis will include tables with all AEs and neurological and systemic complications. A descriptive analysis summarising efficacy variables in each group will be performed and an exploratory analysis will be conducted by comparing both treatment groups using the Wilcoxon rank sum test or Student's t-test.

\section{Plans for predefined subgroup analyses}

1. The 'intention to treat' population will consist of all patients for whom baseline variables and at least 1 value of a principal or secondary variable are available. If a final visit variable is not obtained for a patient, it will be replaced by the last available value $(\mathrm{LOCF}=\mathrm{Last}$ Observation Carried Forward).

2. The 'per protocol' population will consist of all patients for whom infusion of the study medication had been started with no major protocol violation.

3. The 'safety analysis population' will consist of all patients for whom infusion of the study medication had been started.

\section{Data monitoring body}

Coordination, management, monitoring, data management and the statistical analysis will be performed by the SCReN.

\section{Auditing}

During the progress of the study, audit visits may be conducted at the participating centres. The investigator will allow direct access to the source data/documents for monitoring, auditing, review by the ethical research committee and inspection by the health authorities.

\section{Patient and public involvement}

The development of the research objectives and outcomes are based on the neurologist's experience treating this profile of patients and the desire to optimise brain tissue repair therapies. Patients and their advisors were not involved in the design, recruitment or conduct of this study. 


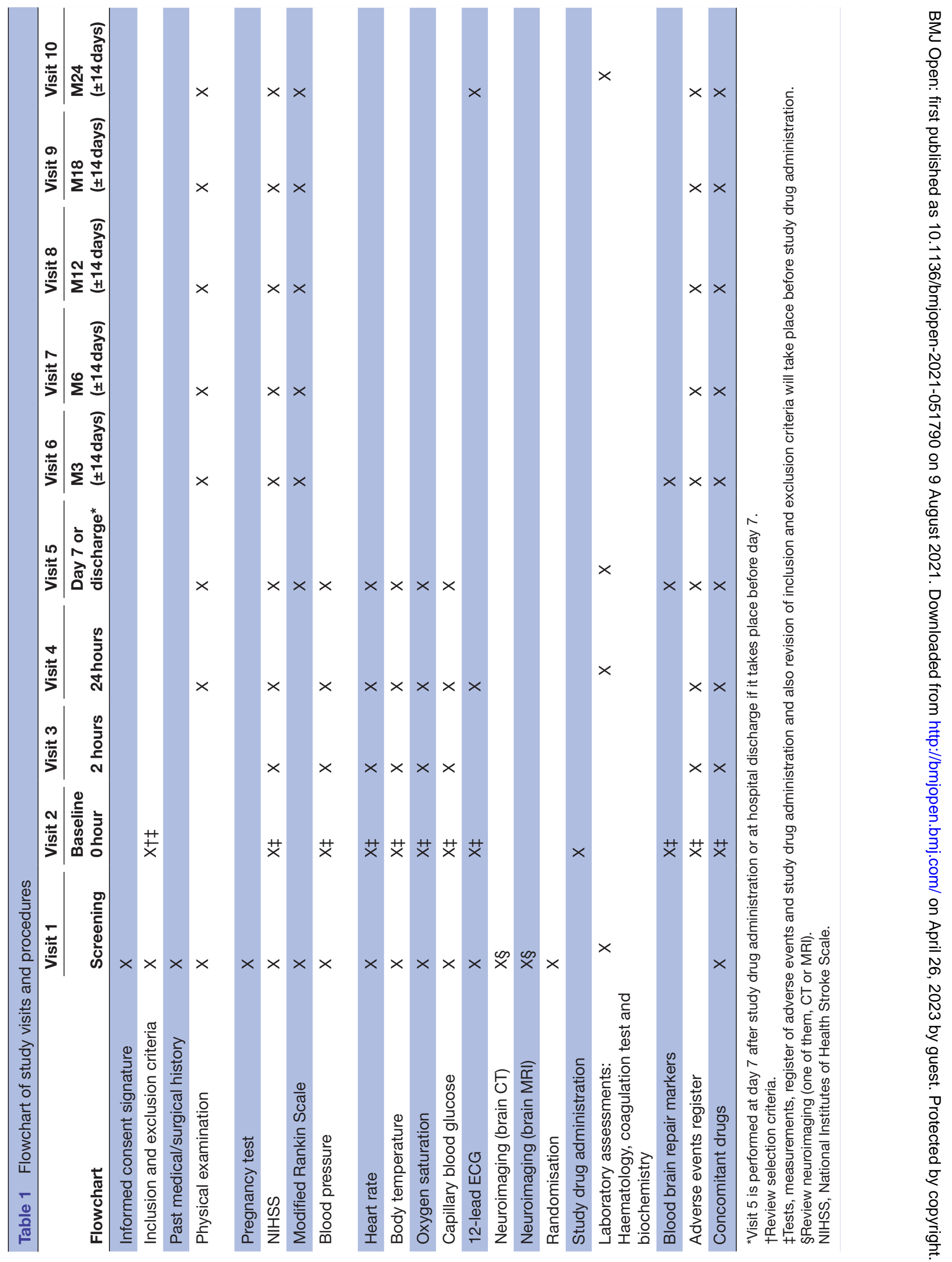




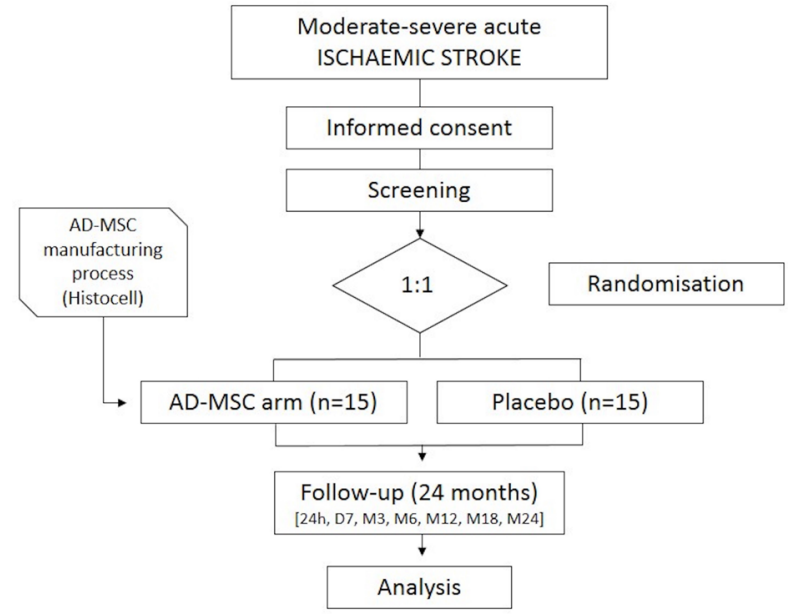

Figure 1 Schematic flowchart of the clinical trial. AD-MSCs, allogeneic adipose tissue-derived mesenchymal stem cells

\section{SUMMARY}

AD-MSCs administered intravenously in the first days from stroke symptom onset could be a safe and promising therapy to enhance recovery from brain ischaemia and to help reduce disability. This type of cell therapy has already demonstrated its efficacy and safety in preclinical research, but further studies are needed to prove similar results in patients with stroke. The advantages of AD-MSCs over other cell types are their accessibility and abundance as well as their low immunogenicity. An intravenous infusion appears to be the most appropriate manner of administration, given it is the least aggressive, and cell therapies can be performed by modification of trophic factors and immunomodulation.

This clinical trial aims to demonstrate that early intravenous administration of AD-MSCs has no major safety complications and that it could possibly reduce disability due to stroke. Also, analysis of blood biomarkers and trophic factors will help elucidate and deepen our understanding of the mechanisms of action of cell therapy, along with previous results from other clinical trials. ${ }^{24} 25$ The results of the AMASCIS-02 trial, together with those from the AMASCIS-01 trial, will increase our knowledge of the safety of intravenous AD-MSC administration in patients with ischaemic stroke. We will then be able to conduct larger clinical trials to evaluate their efficacy and possibly enable their use in clinical practice in the near future.

\section{Ethics and dissemination}

\section{Research ethics approval}

The researchers will adhere strictly to the provisions of this protocol and will complete the case report forms. The study will be performed according to the recommendations for clinical studies and the evaluation of drugs in humans, as contained in the Declaration of Helsinki (revised in successive world assemblies) and in the current Spanish and European legislation on clinical studies and patient data confidentiality. The study will follow the principles of Good Clinical Practice. This study (protocol V.2, dated 5 May 2020) has been approved by the Clinical Research Ethics Committee of La Paz University Hospital (Madrid, Spain) and by the Spanish Agency of Medication and Health Products, and has been registered in Eudra CT (2019-001724-35) and ClinicalTrials. gov (NCT04280003).

\section{Protocol amendments}

All protocol amendments will be evaluated by the Ethics Committee and the Spanish Agency of Medication and Health Products, following the principles of Good Clinical Practice and national legislation. All modifications of the study protocol will be communicated by updating the trial registry at ClinicalTrials.gov.

\section{Consent to participate}

Informed consent will be obtained by a recruiting investigator for all individual participants included in this study, or by their family member/representative (if the patient has aphasia or altered consciousness at that moment). Reconsent from the patient will be obtained whenever possible if initial consent was provided by family members/representatives. The informed consent document is written in Spanish. The informed consent documents are available as online supplemental files.

\section{Consent for publication}

According to the Royal Decree 1090/2015, the results of this clinical trial will be published once the study is completed in an Open Access format.

\section{Confidentiality}

All participant information as well as the electronic database will remain in secure storage at both study centres during the trial and up to 25 years after its finalisation.

\section{Access to data}

All investigators and each centres' monitor will have access to the electronic trial data during the data collection period; after completion of the study, the data will also be accessible to statisticians. Only the blinded study nurses and Histocell will have access to randomisation codes until the data collection period is complete.

\section{Availability of data and material}

Data will be available from the corresponding author on reasonable request, if it is considered compliant with the General Data Protection Regulation and the datasharing agreement is approved by the relevant Spanish authorities. On study finalisation, anonymised individual patient data will be available on a public repository of the Community of Madrid.

\section{Ancillary and post-trial care}

Patients will be treated during and after the clinical trial is completed with the best medical treatments according to the state of the art. Participants will not receive economic benefits beyond compensation from the study insurance company in cases of study treatment-related AEs (Hannover seguros España). 


\section{Dissemination policy}

Output from this study will include journal publications, conference presentations and community reporting. Output will not identify participants.

\section{Author affiliations}

${ }^{1}$ Department of Neurology and Stroke Centre; Neurosciences Area, Hospital La Paz Institute for Health Research-IdiPAZ (La Paz University Hospital-Universidad Autónoma de Madrid), Madrid, Spain

${ }^{2}$ Department of Neurology and Stroke Research Program, Hospital Universitario Virgen del Rocío.Institute of Biomedicine of Seville, IBiS/Hospital Universitario Virgen del Rocío/CSIC/University of Seville, Seville, Spain

${ }^{3}$ Department of Neurology and Stroke Research Program, Hospital Universitario Virgen Macarena,Institute of Biomedicine of Seville, IBiS/Hospital Universitario Virgen del Rocío/CSIC/University of Seville, Seville, Spain

${ }^{4}$ Department of Clinical Pharmacology, Hospital La Paz Institute for Health Research-IdiPAZ (La Paz University Hospital-Universidad Autónoma de Madrid), Madrid, Spain

Contributors The first draft of the manuscript was written by EdC-R, and all authors commented on previous versions of the manuscript. BF, ED-T, FM, JM, AMB and MG-F participated in the study conception and design. All authors have read and approved the final manuscript.

Funding This clinical trial has been promoted by the La Paz University Hospital Institute for Health Research—IdiPAZ (La Paz University Hospital—Universidad Autónoma de Madrid) and sponsored from a competitive grant from the Carlos III Health Institute Healthcare Research Fund, and cofunded by the European Regional Development Fund (ERDF) 'A way to make Europe'/'Investing in your future' (PIC18/00016). This clinical trial has been supported by Plataforma Española de Investigación Clínica y Ensayos Clínicos, SCReN (Spanish Clinical Research Network), funded by Carlos III Health Institute-General Subdirection for Evaluation and Promotion of Research, research PT17/0017/0013; State Plan for Scientific Investigation, Technology and Innovation (2017-2020) and cofunded by European Regional Development Fund/European Social fund 'A way to make Europe'/'Investing in your future' (grant ID PT17/0017/0013).

Disclaimer The Funders and Sponsor will not interfere in the selection processes of patients, analysis of data and/or publication of the results or in any other process that might interfere with the results of the study. Funding will be independent of the results of the study. The principal investigator has ultimate authority over any of these activities.

\section{Competing interests None declared.}

Patient consent for publication Not required.

Provenance and peer review Not commissioned; externally peer reviewed.

Supplemental material This content has been supplied by the author(s). It has not been vetted by BMJ Publishing Group Limited (BMJ) and may not have been peer-reviewed. Any opinions or recommendations discussed are solely those of the author(s) and are not endorsed by BMJ. BMJ disclaims all liability and responsibility arising from any reliance placed on the content. Where the content includes any translated material, BMJ does not warrant the accuracy and reliability of the translations (including but not limited to local regulations, clinical guidelines, terminology, drug names and drug dosages), and is not responsible for any error and/or omissions arising from translation and adaptation or otherwise.

Open access This is an open access article distributed in accordance with the Creative Commons Attribution Non Commercial (CC BY-NC 4.0) license, which permits others to distribute, remix, adapt, build upon this work non-commercially, and license their derivative works on different terms, provided the original work is properly cited, appropriate credit is given, any changes made indicated, and the use is non-commercial. See: http://creativecommons.org/licenses/by-nc/4.0/.

ORCID iD

Blanca Fuentes http://orcid.org/0000-0002-0363-862X

\section{REFERENCES}

1 GBD 2016 Neurology Collaborators. Global, regional, and national burden of neurological disorders, 1990-2016: a systematic analysis for the global burden of disease study 2016. Lancet Neurol 2019;18:459-80.
2 Gutiérrez-Fernández M, Fuentes B, Rodríguez-Frutos B, et al. Trophic factors and cell therapy to stimulate brain repair after ischaemic stroke. J Cell Mol Med 2012;16:2280-90.

3 Bang OY, Kim EH, Cha JM, et al. Adult stem cell therapy for stroke: challenges and progress. J Stroke 2016;18:256-66.

4 Krause M, Phan TG, Ma H, et al. Cell-based therapies for stroke: are we there yet? Front Neurol 2019;10:656.

5 Rodríguez-Frutos B, Otero-Ortega L, Gutiérrez-Fernández M, et al. Stem cell therapy and administration routes after stroke. Trans Stroke Res 2016;7:378-87.

6 Savitz SI. Developing cellular therapies for stroke. Stroke 2015;46:2026-31.

7 Vu Q, Xie K, Eckert M, et al. Meta-analysis of preclinical studies of mesenchymal stromal cells for ischemic stroke. Neurology 2014:82:1277-86

8 Lalu MM, Montroy J, Dowlatshahi D, et al. From the lab to patients: a systematic review and meta-analysis of mesenchymal stem cell therapy for stroke. Trans/ Stroke Res 2020;11:345-64.

9 Sarmah D, Agrawal V, Rane P, et al. Mesenchymal stem cell therapy in ischemic stroke: a meta-analysis of preclinical studies. Clin Pharmacol Ther 2018;103:990-8.

10 Cui L-L, Golubczyk D, Tolppanen A-M, et al. Cell therapy for ischemic stroke: are differences in preclinical and clinical study design responsible for the translational loss of efficacy? Ann Neurol 2019;86:5-16.

11 Xue P, Wang M, Yan G. Mesenchymal stem cell transplantation as an effective treatment strategy for ischemic stroke in Asia: a metaanalysis of controlled trials. Ther Clin Risk Manag 2018;14:909-28.

12 Hess DC, Wechsler LR, Clark WM, et al. Safety and efficacy of multipotent adult progenitor cells in acute ischaemic stroke (MASTERS): a randomised, double-blind, placebo-controlled, phase 2 trial. Lancet Neurol 2017;16:360-8.

13 Gutiérrez-Fernández M, Otero-Ortega L, Ramos-Cejudo J, et al. Adipose tissue-derived mesenchymal stem cells as a strategy to improve recovery after stroke. Expert Opin Biol Ther 2015;15:873-81.

14 Lee SH, Jin KS, Bang OY, et al. Differential migration of mesenchymal stem cells to ischemic regions after middle cerebral artery occlusion in rats. PLoS One 2015;10:e0134920.

15 Chang K-A, Lee J-H, Suh Y-H. Therapeutic potential of human adipose-derived stem cells in neurological disorders. J Pharmacol Sci 2014;126:293-301

16 Gutiérrez-Fernández M, Rodríguez-Frutos B, Otero-Ortega L, et al. Adipose tissue-derived stem cells in stroke treatment: from bench to bedside. Discov Med 2013;16:37-43.

17 Gutiérrez-Fernández M, Rodríguez-Frutos B, Ramos-Cejudo J, et al. Effects of intravenous administration of allogenic bone marrowand adipose tissue-derived mesenchymal stem cells on functional recovery and brain repair markers in experimental ischemic stroke. Stem Cell Res Ther 2013;4:11.

18 Gómez-de Frutos MC, Laso-García F, Diekhorst L, et al. Intravenous delivery of adipose tissue-derived mesenchymal stem cells improves brain repair in hyperglycemic stroke rats. Stem Cell Res Ther 2019;10:1-13.

19 Díez-Tejedor E, Gutiérrez-Fernández M, Martínez-Sánchez P, et al. Reparative therapy for acute ischemic stroke with allogeneic mesenchymal stem cells from adipose tissue: a safety assessment: a phase II randomized, double-blind, placebo-controlled, single-center, pilot clinical trial. J Stroke Cerebrovasc Dis 2014;23:2694-700.

20 Fuentes B, Gutiérrez-Zúñiga R, Gutiérrez-Fernández M, et al. Shortterm safety of allogeneic adipose-tissue derived mesenchymal stem cells in acute ischemic stroke (AMASCIS): a phase II, randomised, double-blind, placebo-controlled, single-centre, pilot clinical trial. SSRN Journal.

21 Hirano A, Sano M, Urushihata N, et al. Assessment of safety and feasibility of human allogeneic adipose-derived mesenchymal stem cells in a pediatric patient. Pediatr Res 2018;84:575-7.

22 Zheng G, Huang L, Tong $\mathrm{H}$, et al. Treatment of acute respiratory distress syndrome with allogeneic adipose-derived mesenchymal stem cells: a randomized, placebo-controlled pilot study. Respir Res 2014;15:39.

23 Álvaro-Gracia JM, Jover JA, García-Vicuña R, et al. Intravenous administration of expanded allogeneic adipose-derived mesenchymal stem cells in refractory rheumatoid arthritis (Cx611): results of a multicentre, dose escalation, randomised, single-blind, placebocontrolled phase Ib/lla clinical trial. Ann Rheum Dis 2017;76:196-202.

24 Moniche F, Gonzalez A, Gonzalez-Marcos J-R, et al. Intra-arterial bone marrow mononuclear cells in ischemic stroke: a pilot clinical trial. Stroke 2012;43:2242-4.

25 Moniche F, Montaner J, Gonzalez-Marcos J-R, et al. Intra-arterial bone marrow mononuclear cell transplantation correlates with GMCSF, PDGF-BB, and MMP-2 serum levels in stroke patients: results from a clinical trial. Cell Transplant 2014;23(Suppl 1):57-64. 